\title{
Vorwort zur 3. Auflage
}

Die Tourismusbranche war seit den 2010er Jahren und bis zum Ausbruch der CoronaPandemie durch eine hohe Dynamik geprägt. Mit dem Ende der Finanzkrise nahm die Anzahl der Reisenden Jahr für Jahr verlässlich zu und die Prognosen der UNWTO gaben keinen Anlass, an der Fortsetzung dieses Trends zu zweifeln. Eine relativ starke Weltwirtschaft, wachsende Mittelklassen in Schwellenländern, aber auch neue technologiegetriebene Geschäftsmodelle sind nur einige Rahmenbedingungen, die den über Jahre anhaltenden Wachstumskurs des Tourismus ermöglichten. Die Tourismuswirtschaft profitierte von dem zunehmenden Wohlstand der Nationen und den ansteigenden Freizeit- und Urlaubreisen. Zudem brachte die Globalisierung eine weltweite Vernetzung der Unternehmen mit sich und förderte den Anstieg von touristischen Geschäftsreisen. Während einerseits die absolute Zahl der Reisenden immer neue Dimensionen erlangte, nahm andererseits die Kritik an Wachstum und der gesamten Tourismusindustrie zu. Neben den positiven Auswirkungen des Tourismus für Ökonomie und Gesellschaft traten vielerorts durch den Tourismus ausgelöste oder verstärkte negative Effekte in den Mittelpunkt des Interesses. Zu den seit langem bekannten Kritikpunkten wurden neue Aspekte hinzugefügt und die unter dem Stichwort „Overtourism“ geführten akademischen und medialen Diskussionen waren im vollen Gange.

Die jähe Unterbrechung durch die globale Covid-19-Pandemie stürzt den internationalen Tourismus voraussichtlich in seine bislang schwerste Krise. Weltweite Reiseverbote, Quarantänemaßnahmen und Social Distancing lassen den touristischen Konsum in kürzester Zeit drastisch einbrechen. Zahlreiche touristische Anbieter ganz unterschiedlicher Größe müssen auf staatliche Hilfen zurückgreifen, sind in ihrer Existenz bedroht oder sind wirtschaftliche Opfer des Virus. Einiges spricht dafür, dass die Landschaft der touristischen Anbieter nach der Krise anders aussehen wird als vorher.

Die touristischen Nachfrager hingegen müssen auf (Urlaubs- oder Geschäfts-) Reisen und den Besuch bestimmter Destinationen verzichten oder neue, durch Sicherheitsmaßnahmen geprägte Reiseerlebnisse und -erfahrungen machen. „Der gegenwärtige Tourist“ muss sich neu orientieren, sich auf neue Rahmenbedingungen und Spielregeln und z. T. auf neue Reiseziele und Reiseformen einlassen. „Der zukünftige Tourist“" wird durch die Erfahrungen mit der Pandemie und deren Folgen geprägt sein.

Einiges spricht aber auch dafür, dass verschiedene Dynamiken des Tourismusmarktes aus der Vor-Corona-Zeit von der Branche wieder aufgenommen werden können - auch wenn sich die Festlegung eines diesbezüglichen Datums gegenwärtig noch verbietet. Wir gehen gleichwohl davon aus, dass der Zeitpunkt kommen wird, und dass vieles, was zuvor zum Wissenspool des modernen Touristikers gehörte, nach wie vor auch dann noch von Relevanz sein wird. Hieraus speist sich unsere Zuversicht, dass das vorliegende Werk - trotz der unmittelbar nach Redaktionsschluss ausbre- 
chenden Covid-19-Pandemie - seinen Zweck erfüllen kann. Eine umfassende Integration der aktuellen und noch anstehenden Folgen der Pandemie bleibt der vierten Auflage vorbehalten.

Im Fokus der hier vorliegenden dritten Auflage steht eine umfassende wissenschaftliche, besonders auch wirtschaftliche Betrachtung des Tourismus. Die beiden vorherigen Auflagen waren stark durch die Angebotsseite des Tourismus geprägt, wobei v. a. auf die Themengebiete Verkehr, Hotellerie, Reisemittler und -veranstalter sowie Destinationen eingegangen wurde. Diese Kerngebiete des angebotsorientierten Tourismus finden sich in aktualisierter Form auch im vorliegenden Werk wieder. Doch wurde die dritte Auflage unseres Lehrbuches deutlich erweitert, indem wir sieben weitere Kapitel neu konzipiert und integriert haben: Grundlagen, touristische Nachfrage, Tourismusmanagement, Informationsmanagement, Geschäftsreisen, Tourismuspolitik sowie Tourismuskritik. Vor diesem Hintergrund handelt es sich eher um eine Neukonzeption als um eine Fortschreibung der 2. Auflage. Wir wünschen allen Leserinnen und Lesern sowohl viele gewinnbringende Erkenntnisse als auch viel Freude mit dem so entstandenen Lehrbuch.

Kempten, Heide, Deggendorf und Wilhelmshaven im Mai 2020

Axel Schulz

Bernd Eisenstein

Marco A. Gardini

Waldemar Berg

Torsten H. Kirstges 